\title{
Fracturas atípicas de fémur proximal asociadas al uso prolongado de bifosfonatos
}

\author{
Yamile V. Neder, Alejandro Fazio, Pablo Valle, \\ Facundo Faule, Mariano Finola \\ Servicio de Ortopedia y Traumatología, \\ Hospital Privado Universitario de Córdoba, Córdoba
}

\section{RESUMEN}

Introducción: Los bifosfonatos constituyen el tratamiento de primera elección de la enfermedad osteoporótica. Algunos efectos adversos ponen en duda su uso prolongado, como las fracturas atípicas de fémur proximal. Los objetivos de este estudio fueron determinar la relación entre consumo de bifosfonatos y fracturas atípicas de fémur, y mostrar la incidencia en nuestra institución.

Materiales y Métodos: Estudio retrospectivo, observacional, análisis de caso-control no pareado. Se incluyeron pacientes $>55$ años, con fractura de fémur que ingresaron entre el 1 de enero de 2009 y el 31 de mayo de 2015. Las variables consideradas fueron: sexo, edad, tipo de fractura; uso, tipo y tiempo de consumo de bifosfonatos. Las fracturas se distribuyeron en típicas: pertrocantéricas, y cuello femoral, y atípicas: subtrocantéricas y diafisarias. Se consideraron como casos las fracturas atípicas y como controles, las pertrocantéricas.

Resultados: Se incluyó a 517 pacientes que cumplieron los criterios de inclusión. Cuarenta y dos fracturas eran atípicas y 236, típicas. Hubo predominio del sexo femenino ( $81,4 \%$ en los casos y $83 \%$ en los controles). La edad promedio fue de 76 y 80 años, respectivamente. La asociación con bifosfonatos fue del 44,2\% en los casos y 15,3\% en los controles (11,6\% y $0,8 \%$ en las fracturas pertrocantéricas, respectivamente).

Conclusiones: El alendronato se asoció significativamente con fracturas atípicas de fémur. No se halló relación con el tiempo de consumo; sin embargo, la incidencia fue más alta luego de 4.5-5 años de consumo.

Palabras clave: Fractura de cadera; osteoporosis; bifosfonatos.

Nivel de Evidencia: IV

\section{ATYPICAL FRACTURES OF PROXIMAL FEMUR ASSOCIATED WITH PROLONGED USE OF BISPHOSPHONATES}

\begin{abstract}
Introduction: Bisphosphonates are currently considered a first-choice treatment for osteoporotic disease. A number of adverse effects call into question its long-term administration, such as proximal femoral pathological fractures. The purposes of this study were to determine the relationship between consumption of bisphosphonates and atypical femoral fractures, and to report the incidence in our institution.

Methods: Retrospective, observational study, case-control unpaired analysis. Patients $>55$ years old with femoral fractures admitted from January 1, 2009 to May 31, 2015 were included. Considered variables were: sex, age, type of fracture, use, type and time of consumption of bisphosphonates. Fractures were divided into typical: pertrochanteric, and femoral neck, and atypical: subtrochanteric and diaphyseal. Atypical fractures were cases, and typical fractures functioned as controls.
\end{abstract}

Conflicto de intereses: Los autores no declaran conflictos de intereses. 
Results: A total of 517 patients met the inclusion criteria and were included in the study. Forty-two fractures were atypical and 236 were typical. Female sex predominated ( $81.4 \%$ of cases, and $83 \%$ of controls). Average age was 76 and 80 years, respectively. Association with bisphosphonates was observed in $44.2 \%$ of the cases, and $15.3 \%$ of controls (11.6\% and $0.8 \%$ in pertrochanteric fractures, respectively).

Conclusions: Alendronate was significantly associated with atypical femoral fractures. A relationship with length of consumption was not detected; however, incidence was higher after 4.5-5 years of use.

Key words: Hip fracture; osteoporosis; bisphosphonates. Level of Evidence: IV

\section{Introducción}

En la actualidad, los bifosfonatos son considerados el tratamiento de primera elección para la enfermedad osteoporótica. Su reconocida eficacia antifractura en todos los tipos de osteoporosis y su presencia en el mercado desde hace varios años, los convierten en los fármacos de uso más extendido. Una serie de efectos adversos relacionados han encendido la voz de alarma en el tratamiento continuado a largo plazo. Entre estos se describen las fracturas atípicas principalmente de fémur proximal consideradas como tales por su localización y apariencia radiológica que no cumplen el clásico perfil de la fractura por fragilidad (enfermedad osteoporótica). ${ }^{1}$ La prolongada inhibición del remodelado óseo podría ser el sustento fisiopatológico para su explicación, aunque todavía no está claramente establecida esta relación causal.

Los objetivos de este estudio fueron determinar la relación entre el consumo crónico de bifosfonatos y las fracturas atípicas de fémur, evaluar la relación entre el tiempo de consumo de bifosfonatos y el evento de fractura, y proponer un algoritmo de seguimiento en el paciente con consumo crónico de bifosfonatos a fin de detectar casos con potencial riesgo de fractura atípica. También se llevó a cabo una revisión bibliográfica.

\section{Materiales y Métodos}

Estudio retrospectivo, observacional, análisis de caso control no pareado. Se incluyeron pacientes sin distinción de sexo, $>55$ años, con diagnóstico de fractura de cadera/ fémur proximal que ingresaron en el Servicio de Ortopedia y Traumatología del Hospital Privado Universitario de Córdoba, entre el 1 de enero de 2009 y el 31 de mayo de 2015. Las variables evaluadas fueron: sexo, edad, tipo de fractura, uso de bifosfonatos, tipo y tiempo de consumos, tipo de traumatismo, comorbilidades, fármacos asociados y diagnóstico de osteoporosis por densitometría ósea. Las fracturas se distribuyeron según la localización del trazo en: Típicas: laterales de cadera (pertrocantéricas), y Mediales y Atípicas: subtrocantéricas y diafisarias de fémur del tercio proximal, medial o distal con antecedente de trauma de baja energía o espontáneas.
Los criterios de exclusión fueron edad $<55$ años, fractura de alto impacto en el contexto de politraumatismos, fracturas pelvianas y fracturas periprotésicas. Para el análisis se tomaron como casos las fracturas atípicas y como controles las fracturas típicas. Las variables se resumieron en frecuencia, media y desviación estándar (DE). Se realizaron análisis bivariado, multivariado, prueba t y con regresión logística. Se consideró significativo un valor $\mathrm{p}<0,05$ con intervalo de confianza del $95 \%$.

\section{Resultados}

Se revisaron 767 historias clínicas electrónicas de pacientes con fractura de cadera (período de 6 años y 5 meses), 517 cumplieron con los criterios de inclusión. Las fracturas eran: laterales $(45,7 \%, \mathrm{n}=236)$, mediales $(43,3 \%, \mathrm{n}=224)$, diafisarias proximal y medial $(4,6 \%$, $\mathrm{n}=24)$, subtrocantéricas $(3,7 \%, \mathrm{n}=19)$ y diafisarias distal $(2,7 \%, \mathrm{n}=14)$.

En el análisis de caso/control quedaron 279 fracturas: 43 atípicas y 236 típicas. Predominó el sexo femenino: $81,4 \%$ (35/43) en los casos y $83 \%$ (196/236) en los controles. La edad fue de 76 años (DE 9.3) y 80 años (DE 8.7), respectivamente $(\mathrm{p}=0,00)$ (cociente de posibilidades $[$ odds ratio, $\mathrm{OR}]=0,9)$. En el análisis bivariado, la asociación con bifosfonatos fue del 44,2\% (19/43) en los casos y $15,3 \%(36 / 235)$ en los controles $(p=0,00)$ y se mantuvo en el análisis multivariado con un OR de 3,6. El alendronato fue el único tipo de bifosfonato que tuvo una asociación significativa con las fracturas atípicas: $11,6 \%$ $(5 / 43)$ y $0,8 \%(2 / 235)$ en las típicas $(p=0,00)$. Las fracturas atípicas fueron predominantemente no traumáticas $(53,5 \%, 23 / 43)$ frente al 9,3\% (22/235) en las típicas, con una significancia estadística $(p=0,000)$. En el análisis bivariado de comorbilidades, se asoció a las fracturas atípicas con osteoporosis $(55,8 \%, 24 / 43$ de los casos y $17 \%$, $40 / 235$ de los controles) $(\mathrm{p}=0,00)$, con enfermedad autoinmune $(9,3 \%, 4 / 43$ y $1,7 \%, 4 / 236$, respectivamente) $(\mathrm{p}=0,00)$ y con el uso de corticoides $(18,6 \%, 8 / 43$ y $5,5 \%, 13 / 236$, respectivamente) $(\mathrm{p}=0,003)$.

Al realizar el análisis multivariado, sólo se mantuvo la asociación de fractura atípica con osteoporosis (OR 5,5). El 43\% (18/42) de los casos y el 17\% (38/229) de los con- 
troles tenían una densitometría ósea $(\mathrm{p}=0,00)$. No se encontraron diferencias entre los años de uso de bifosfonatos $(5,11$ en fracturas atípicas y 4,41 en las típicas; $p=0,08)$.

\section{Discusión}

Los bifosfonatos son fármacos antiosteoporóticos altamente eficaces en la prevención de fracturas vertebrales y no vertebrales. La incidencia de fracturas atípicas de fémur proximal en los pacientes tratados con bifosfonatos es relativamente baja. La reducción del riesgo de fractura oscila entre el $40 \%$ y el $70 \%$ para la fractura vertebral y del $40 \%$ al $50 \%$ para la fractura de fémur. ${ }^{2}$ De entre todas las complicaciones descritas, ha sido la progresiva aparición de casos de fracturas atípicas de fémur la que ha puesto en alerta y cuestionado el tratamiento a largo plazo. Desde el punto de vista fisiopatológico, el tratamiento prolongado conlleva una supresión excesiva del remodelado óseo, situación en la que existiría mayor mineralización, acumulación de hueso envejecido sin remodelar y, por lo tanto, un mayor riesgo de microfracturas. Sin embargo, no se puede establecer una relación causal entre el tratamiento prolongado con bifosfonatos y la aparición de fracturas atípicas, es probable que estos fármacos jueguen un papel en su desarrollo sin que esta sea la única condición.

La incidencia de fracturas femorales atípicas se incrementa con el tiempo de exposición a los bifosfonatos. Pueden ser unilaterales o bilaterales, incompletas o completas, se caracterizan por un trazo habitualmente transversal o ligeramente oblicuo, no conminuto y con formación de una característica espícula medial. En la mayoría de los casos, se puede apreciar un engrosamiento cortical lateral. Ante un paciente que recibe tratamiento prolongado con bifosfonatos y refiere dolor en la ingle o en el muslo de forma continuada, sin antecedentes de trauma de alta energía y, en todos los casos de fractura atípica unilateral completa e incompleta, con síntomas o sin ellos, se recomienda tomar una radiografía simple anteroposterior/lateral del fémur contralateral que incluya la totalidad de la diáfisis. ${ }^{3}$ Si este estudio no resulta totalmente concluyente y el nivel de sospecha clínica es elevado estaría indicada una tomografía computarizada, una resonancia magnética o una gammagrafía del fémur contralateral en busca de signos predictivos de fractura por insuficiencia o de estrés en evolución (Figura 1).

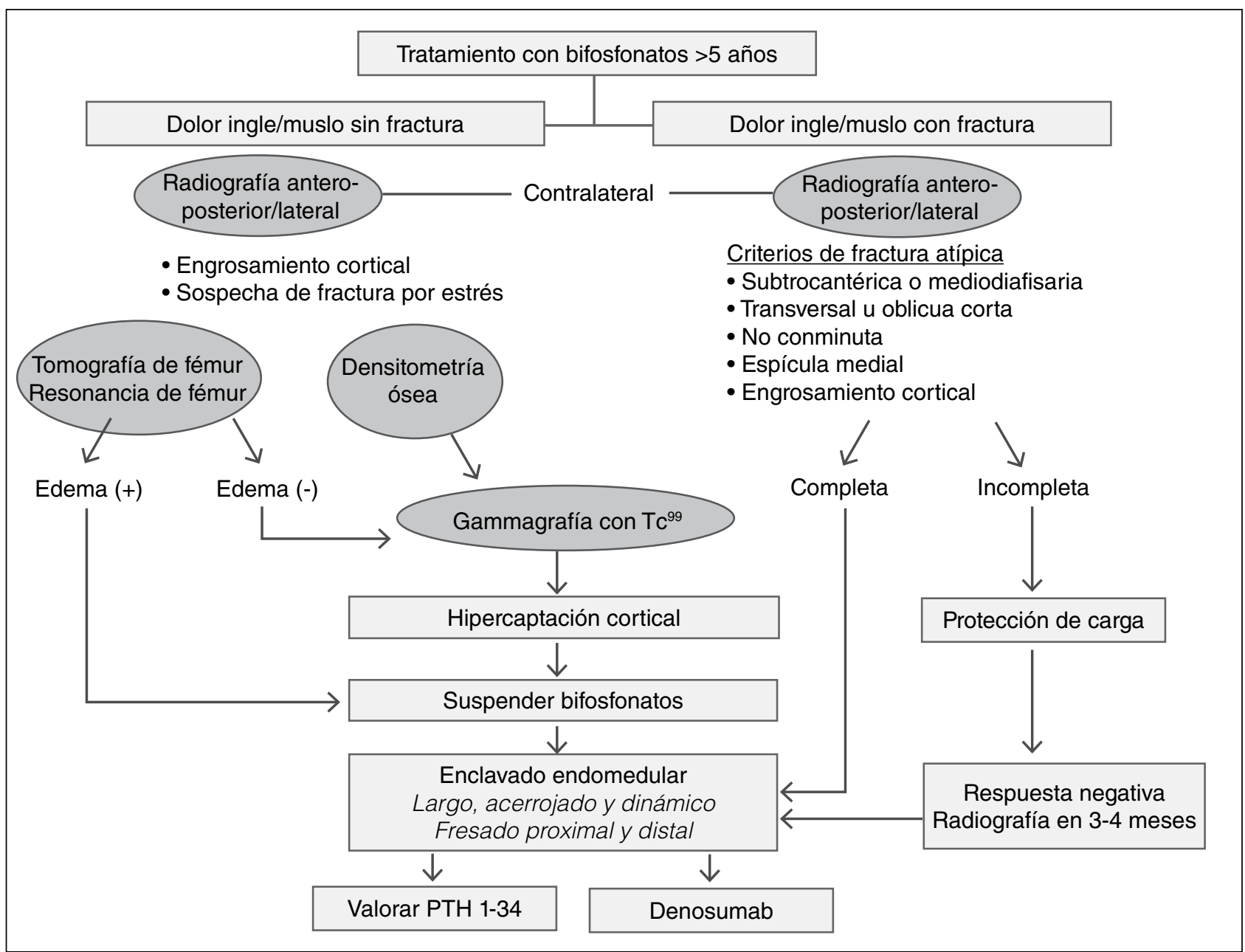

Figura 1. Algoritmo de diagnóstico y tratamiento de las fracturas atípicas relacionadas con el uso prolongado de bifosfonatos. 
Estas fracturas requieren una osteosíntesis eficaz mediante enclavado intramedular con clavo largo fresado que proteja de una refractura a toda la diáfisis femoral. En las fracturas atípicas incompletas con dolor en el muslo, sobre todo, si no han respondido al tratamiento conservador, podría estar recomendado el enclavado endomedular profiláctico.

Actualmente se estima que la incidencia general de las fracturas subtrocantéricas de fémur o mediodiafisarias asociadas a traumatismo de baja energía es del 2-4\% de todas las fracturas de cadera. El $75 \%$ de este tipo de fracturas estaría normalmente relacionado con traumatismos de alta energía. Su repercusión en cuanto a morbimortalidad es similar con tasas de mortalidad del $14 \%$ a los 12 meses y del $25 \%$ a los 24 meses, y con repercusiones funcionales que hacen que la mayoría de los pacientes (71\%) no puedan reanudar las actividades básicas de la vida diaria en condiciones similares a las que tenían antes de la fractura.

Cuando se evalúa más específicamente la epidemiología de las fracturas subtrocantéricas por insuficiencia o estrés, que se producen en determinadas condiciones metabólicas óseas por una deficiente resistencia elásticoplástica del hueso, se constata una prevalencia cercana al $1 \%$ para este tipo de fracturas que se mantiene constante en el tiempo. Se estima que aproximadamente un $25 \%$ de todas las fracturas subtrocantéricas y diafisarias de fémur presentan características que las pueden definir como atípicas relacionadas con el uso prolongado de bifosfonatos.

Por tanto, la incidencia de fracturas atípicas relacionadas con el uso prolongado de bifosfonatos parece ser muy baja, tanto en comparación directa con el número de fracturas subtrocantéricas o diafisarias de fémur en general, como indirecta en relación con el número de fracturas vertebrales, no vertebrales y de cadera prevenidas por este tipo de fármacos. Sin embargo, más allá de los datos que arroja la bibliografía más actual sobre el uso crónico de bifosfonatos y el riesgo de fracturas atípicas, en nuestra institución, hubo un $8,3 \%$ de fracturas atípicas, de las cuales el 11,6\% se asoció al uso crónico de bifosfonatos (>4 años). ${ }^{4}$

En cuanto a las indicaciones y prescripción del uso de bifosfonatos para tratar la enfermedad osteoporótica, se han tenido en cuenta los protocolos diagnósticos basados en resultados de la densitometría ósea, para lo cual la Organización Mundial de la Salud plantea como estándar de referencia diagnóstica un $T$-score inferior a $-2,5$ y la determinación de factores de riesgo en el contexto de los antecedentes personales patológicos. ${ }^{5}$ Estos criterios se basan en las últimas Guías 2012 para el Diagnóstico, Prevención y Tratamiento de la Osteoporosis elaboradas por la Asociación Argentina de Osteología y Metabolismo Mineral, y la Sociedad Argentina de Osteoporosis.

En la actualidad, a tenor de la bibliografía disponible, los bifosfonatos continúan siendo el tratamiento estándar de la osteoporosis. La decisión de iniciar o no un tratamiento para la osteoporosis con bifosfonatos debe ser absolutamente individualizada y basarse en una correcta evaluación de la relación riesgo/beneficio que conlleva. Si bien parece claro que los pacientes tratados con bifosfonatos se benefician de una importante reducción del riesgo de fractura durante, al menos, cinco años, el uso continuado debe ser revaluado anualmente. Para aquellos pacientes en los que el riesgo de fractura siga siendo moderadamente elevado, se debe considerar firmemente la continuación del tratamiento. Sin embargo, los pacientes de bajo riesgo, en quienes no se constate una fractura reciente o una osteoporosis densitométrica $(T$-score $>2,5)$ después del curso terapéutico inicial podrían beneficiarse de unas "vacaciones terapéuticas". 6

Se requieren estudios prospectivos bien diseñados para establecer realmente el riesgo de padecer este tipo de fracturas y su posible relación con este tratamiento. Es necesario crear códigos diagnósticos específicos para facilitar su registro, promover su estudio y establecer un adecuado manejo médico-quirúrgico.

Además, se debe realizar un despistaje completo de las osteoporosis secundarias con evaluación del metabolismo fosfocálcico, prestando especial atención y corrigiendo los déficits de vitamina D mediante una adecuada suplementación. En la bibliografía, se destacan las múltiples opciones de tratamiento alternativo antiosteoporótico, además de terapias para mantener niveles normales de calcio y vitamina $\mathrm{D}$; se basan principalmente en terapias con teriparatida con alto costo de adquisición y tratamientos con anticuerpos monoclonales, como denosumab. ${ }^{7}$

\section{Conclusiones}

La indicación de iniciar tratamiento con bifosfonatos resulta de una compleja ponderación de su relación costobeneficio basada en las estimaciones de los factores de riesgo.

El alendronato es el único bifosfonato que se asoció, de manera significativa, con las fracturas atípicas de fémur proximal. No se encontró relación en el tiempo de consumo, probablemente debido a la falta de estos datos en la historia clínica electrónica; sin embargo, se demuestra una tendencia mayor al sobrepasar los 4.5-5 años de consumo.

Se estima que la reducción del riesgo de fracturas no vertebrales por alendronato en pacientes sin fractura previa es del $30 \% .^{8}$ Luego de cinco años de tratamiento, su continuación por otros cinco años redujo la incidencia de fracturas vertebrales en un $45 \%$ comparado con los pacientes que habían recibido el tratamiento activo, pero que no lo continuaron. La reducción absoluta del riesgo fue del $2,4 \%$ debido al escaso número de casos con fracturas. 
Ante la inexistencia de un protocolo de seguimiento de paciente con uso crónico de bifosfonatos, este estudio muestra un algoritmo de medidas terapéuticas y controles bioquímicos e imagenológicos para detectar a tiempo pacientes en riesgo de sufrir fracturas atípicas (Figura 2). Sin embargo, siguiendo la revisión bibliográfica, aún se desconoce la fisiopatogenia responsable de esta asociación patológica como reacción adversa.

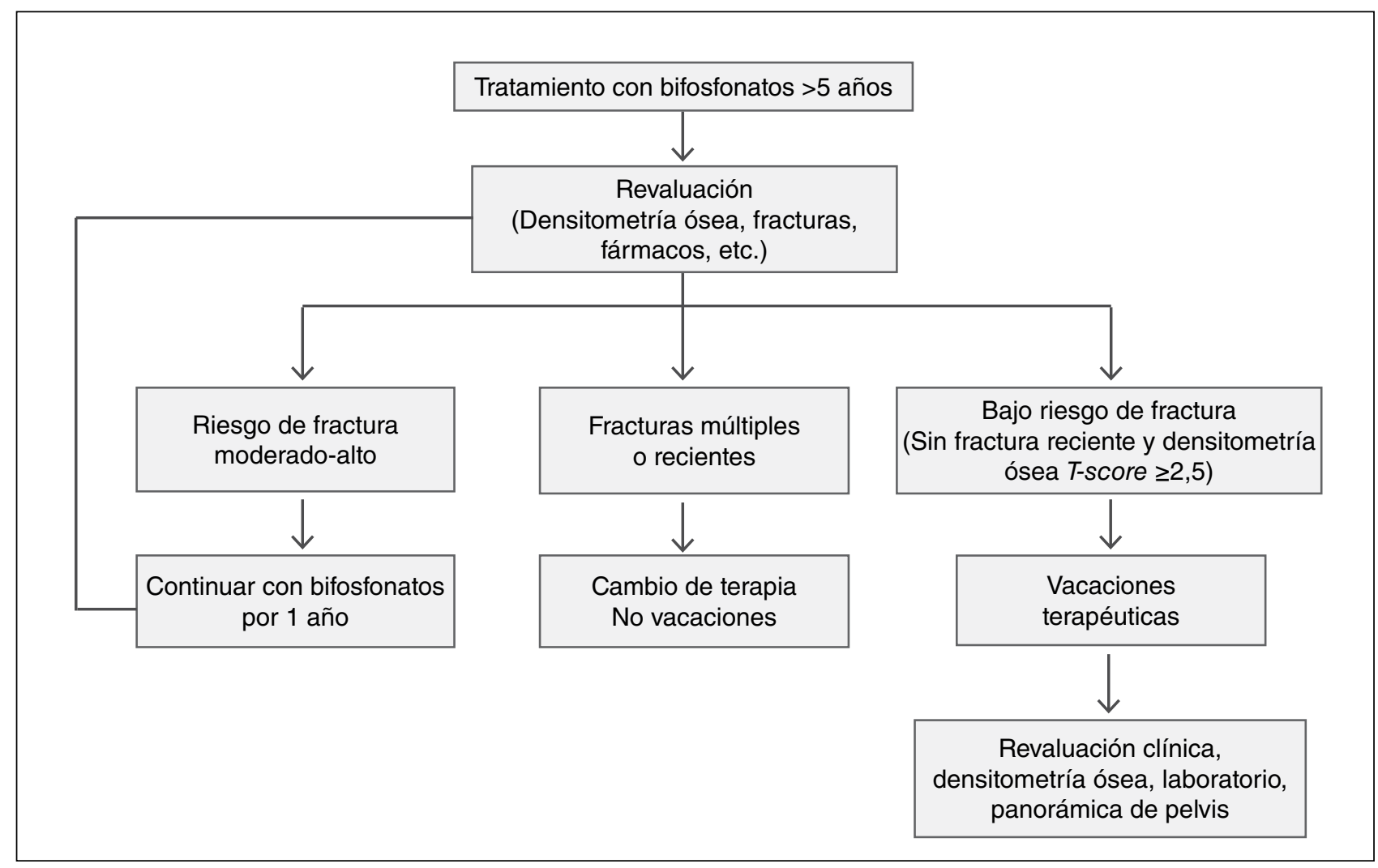

Figura 2. Algoritmo de seguimiento del tratamiento con bifosfonatos.

\section{Bibliografía}

1. Shane E, Burr D, Ebeling PR, Abrahamsen B, Adler RA, Brown TD, et al. Atypical subtrochanteric and diaphyseal femoral fractures: report of a task force of the American Society for Bone and Mineral Research. J Bone Miner Res 2010;25:2267-9.

2. European Medicines Agency. European Medicines Agency concludes class review of bisphosphonates and atypical fractures. Disponible en: http://www.ema.europa.eu/ema/index.jsp?curl=pages/medicines/human/public healh_alerts/2011/04/human_pha_ detail_000027.jsp\&mid=WC0b01ac058001d126.

3. Black DM, Reid IR, Boonen S, Bucci-Rechtweg C, Cauley JA, Cosman F, et al. The effect of 3 versus 6 years of zoledronic acid treatment of osteoporosis: A randomized extension to the HORIZON-Pivotal Fracture Trial (PFT). J Bone Miner Res 2012; 27:243-54.

4. Black DM, Schwartz AV, Ensrud KE, Cauley JA, Levis S, Quandt SA, et al. Effects of continuing or stopping alendronate after 5 years of treatment: the Fracture Intervention Trial Long-term Extension (FLEX): a randomized trial. JAMA 2006;296(24):2927-38.

5. Gedmintas L, Solomon DH, Kim SC. Bisphosphonates and risk of subtrochanteric, femoral shaft, and atypical femur fracture: a systematic review and meta-analysis. J Bone Miner Res 2013;28(8):1729-37.

6. McClung M, Harris ST, Miller PD, Bauer DC, Davison KS, Dian L, et al. Bisphosphonate therapy for osteoporosis: benefits, risks, and drug holiday. Am J Med 2013;126:13-20.

7. Whitaker M, Guo J, Kehoe T, Benson G. Bisphosphonates for osteoporosis--where do we go from here? N Engl J Med 2012;366: 2048-51.

8. Black DM, Bauer DC, Schwartz AV, Cummings SR, Rosen CJ. Continuing bisphosphonate treatment for osteoporosis--for whom and for how long? N Engl J Med 2012;366:2051-3. 